PROCEEDINGS OF THE

AMERICAN MATHEMATICAL SOCIETY

Volume 125, Number 3, March 1997, Pages 723-730

S $0002-9939(97) 03872-0$

\title{
HEIGHT OF FLAT TORI
}

\author{
PATRICK CHIU
}

(Communicated by Dennis A. Hejhal)

\begin{abstract}
Relations between the height and the determinant of the Laplacian on the space of $n$-dimensional flat tori and the classical formulas of Kronecker and Epstein are established. Extrema of the height are shown to exist, and results for a global minimum for 2-d tori and a local minimum for 3-d tori are given, along with more general conjectures of Sarnak and Rankin.
\end{abstract}

\section{Definitions AND NOTATION}

The height of a compact Riemannian manifold $M$ with smooth metric $\sigma$ is the isospectral invariant defined by

$$
h(M, \sigma)=Z^{\prime}(0),
$$

where $Z(s)$ is the zeta-regularization of the determinant of the Laplacian. More precisely, let $0=\lambda_{0} \leq \lambda_{1} \leq \lambda_{2} \leq \cdots$ be the eigenvalues of $M$ with respect to the Laplacian, then the determinant of the Laplacian is defined by $\operatorname{det}^{\prime} \Delta=\prod_{\lambda_{j} \neq 0} \lambda_{j}$, and the zeta-regularization by

$$
Z(s)=\sum_{\lambda_{j} \neq 0} \lambda_{j}^{-s} .
$$

These are formally related through the identity $\operatorname{det}^{\prime} \Delta=\prod_{\lambda_{j} \neq 0} \lambda_{j}=e^{-Z^{\prime}(0)}$. In general, $Z(s)$ has meromorphic continuation to the whole complex plane and is regular at $s=0$, see Sarnak [Sa].

Now let $M$ be an $n$-dimensional torus and $\sigma$ the flat metric, with the volume of $M$ normalized to 1 . Since such a torus can be viewed as $\mathbf{R}^{n}$ modulo a lattice of full rank and determinant 1 , we see that the height is a function on lattices. Let $\mathbf{L}^{n}$ denote this space of lattices, and $\mathbf{H}^{n}$ be the upper-half plane defined by $\operatorname{GL}(n, \mathbf{R}) / O(n) Z$ where $Z$ is the center of $\operatorname{GL}(n, \mathbf{R})$. Then as is well known, we may identify $\mathbf{L}^{n}$ with $\mathrm{SL}(n, \mathbf{Z}) \backslash \mathbf{H}^{n}$.

For a lattice $L \in \mathbf{L}^{n}$ with basis $v_{1}, \ldots, v_{n} \in \mathbf{R}^{n}$, we associate to it a matrix

$$
V=\left(\begin{array}{c}
v_{1} \\
\vdots \\
v_{n}
\end{array}\right)
$$

Received by the editors October 15, 1995.

1991 Mathematics Subject Classification. Primary 11M36; Secondary 11F20, 11E45, 11H50, $11 \mathrm{H} 55$.

(C)1997 American Mathematical Society 
and a positive definite quadratic form $Q=V V^{t}$. The dual of a lattice $L$ is the lattice $L^{*}=\{y \mid x \cdot y \in \mathbf{Z}$ for all $x \in L\}$, which has the corresponding matrix $V^{*}=\left(V^{-1}\right)^{t}$ and quadratic form $Q^{*}=Q^{-1}$.

\section{EXPLICIT FORMULAS}

For $n=2$, we have the following formula for the height, see Osgood-PhillipsSarnak [OPS]. Let $M$ be parametrized by $z=x+i y$ with $y>0$ in $S L(2, \mathbf{Z}) \backslash \mathbf{H}^{2}$. The quantity $Z^{\prime}(0)$ is essentially given by Kronecker's Limit Formula, which is defined below in (2.4). In the present case,

$$
k_{2}(z)=C_{2}-\log \left(y|\eta(z)|^{4}\right),
$$

where $C_{2}=2(\gamma-\log 2)$ with $\gamma$ being Euler's constant, and $\eta(z)$ is the Dedekind eta-function. Then the height of a 2 -d flat torus of area 1 is

$$
h(M)=h(z)=\pi^{-1} k_{2}(z)+2 \log 2 .
$$

For $n=3$, we employ GL(3) analogs of Kronecker's Limit Formula and the eta-function, see Efrat [Ef]. A 3-d torus may be parametrized by the Iwasawa decomposition

$$
\tau=M=\left(\begin{array}{ccc}
y_{1} y_{2} & y_{1} x_{2} & x_{3} \\
& y_{1} & x_{1} \\
& & 1
\end{array}\right),
$$

where $x_{1}, x_{2}, x_{3}, y_{1}, y_{2} \in \mathbf{R}$ with $y_{1}, y_{2}>0$. Also, let $x_{4}=x_{3}-x_{1} x_{2}$ and $z_{k}=$ $x_{k}+i y_{k}$ for $k=1,2$.

An analog of the eta function for GL(3) is

$$
\begin{aligned}
g(\tau)=\exp & \left(-\frac{y_{1}^{1 / 2} y_{2}}{8 \pi} E^{*}\left(z_{1}, \frac{3}{2}\right)\right) \\
& \times \prod_{(m, n)}^{\prime}\left|1-\exp \left(-2 \pi y_{2}\left|n z_{1}-m\right|+2 \pi i\left(m x_{2}+n x_{4}\right)\right)\right|,
\end{aligned}
$$

where the product is taken over all nonzero $(m, n) \in \mathbf{Z}^{2}$ modulo \pm 1 .

For Kronecker's Limit Formula, an analog in GL(3) is

$$
k_{3}(\tau)=C_{3}-\frac{2}{3} \log \left(y_{1} y_{2}^{2}\right)-4 \log g(\tau),
$$

where $C_{3}$ is some constant.

Then the height of a $3-d$ flat torus $M$ of volume 1 is given by

$$
h(M)=h(\tau)=(2 \pi)^{-1} k_{3}(z)+2 .
$$

We proceed to derive a formula for the height in arbitrary dimension. For the moduli space of $n$-dimensional flat tori of volume 1 , the eigenfunctions of a torus $M$ are the exponentials and its eigenvalues are given by $Q^{*}[x]$ for $x \in \mathbf{Z}^{n}$. Hence, the zeta-regularization is

$$
Z(M, s)=(2 \pi)^{-2 s} \sum_{x \in \mathbf{Z}^{n}}^{\prime} Q^{*}[x]^{-s}, \quad \operatorname{Re} s>n / 2 .
$$


The sum on the right-hand side is essentially an Epstein zeta-function. For a positive definite quadratic form $Q[x]$, its associated Epstein zeta-function is defined by

$$
Z_{Q}(s)=\sum_{x \in \mathbf{Z}^{n}}^{\prime} Q[x]^{-s}, \quad \operatorname{Re} s>n / 2 .
$$

It has meromorphic continuation and functional equation, e.g. see Terras [Te1]:

Theorem 2.1 (Epstein). Let $Q$ be the matrix of a positive definite quadratic form in $n$ variables. Then $Z_{Q}(s)$ possesses the following properties:

(i) $Z_{Q}(s)$ has meromorphic continuation to the entire complex plane except for a simple pole at $s=n / 2$ with residue $|Q|^{-1 / 2} \pi^{n / 2} \Gamma(n / 2)^{-1}$.

(ii) It satisfies the functional equation

$$
\pi^{-s} \Gamma(s) Z_{Q}(s)=|Q|^{-1 / 2} \pi^{-(n / 2-s)} \Gamma(n / 2-s) Z_{Q^{-1}}(n / 2-s) .
$$

Next we state a generalization of Kronecker's Limit Formula. It gives the constant term in the Laurent expansion of $Z_{Q}(s)$ at the pole $s=n / 2$, which is defined by

$$
k_{n}(Q)=\lim _{s \rightarrow n / 2}\left\{Z_{Q}(s)-\frac{|Q|^{-1 / 2} \pi^{n / 2} \Gamma(n / 2)^{-1}}{s-n / 2}\right\} .
$$

Employing the notation in Terras [Te2], we first decompose $Q$ into block matrix form

$$
Q=\left(\begin{array}{cc}
1 & R^{t} \\
0 & I
\end{array}\right)\left(\begin{array}{cc}
q & 0 \\
0 & Q_{n-1}
\end{array}\right)\left(\begin{array}{cc}
1 & 0 \\
R & I
\end{array}\right),
$$

where $Q_{n-1}$ and $R$ are $(n-1) \times(n-1)$ matrices. Set

$$
z\{b\}=b^{t} R+i \sqrt{q\left(Q_{n-1}^{-1}[b]\right)},
$$

and

$$
c_{n}= \begin{cases}\gamma-\log 2-\frac{1}{2} \sum_{r=1}^{n / 2-1} r^{-1} & \text { for } n \text { even } \\ \gamma-\sum_{r=0}^{(n-3) / 2}(2 r+1)^{-1} & \text { for } n \text { odd }\end{cases}
$$

Then a generalization of Kronecker's Limit Formula derived by Epstein is:

Theorem 2.2 (Kronecker's Limit Formula).

$$
\begin{aligned}
k_{n}(Q)= & Z_{Q_{n-1}}(n / 2)+\pi^{n / 2}\left|Q_{n-1}\right|^{-1 / 2} \Gamma(n / 2)^{-1} \\
& \times\left\{c_{n}-\log \left(q^{1 / 2}\left|\prod_{b \in \mathbf{Z}^{n-1} / \pm 1}^{\prime}(1-\exp (2 \pi i z\{b\}))\right|^{2}\right)\right\} .
\end{aligned}
$$

We now relate $k_{n}(Q)$ to the height $Z^{\prime}(M, 0)$.

Theorem 2.3. Let $M$ be an n-dimensional flat torus of volume 1 , and $Q$ its associated quadratic form. Then the height of $M$ is

$$
h(M)=Z^{\prime}(M, 0)=\pi^{-n / 2} \Gamma(n / 2) k_{n}(Q)+\psi(n / 2)-\psi(1 / 2),
$$

where $\psi(s)=\Gamma^{\prime}(s) / \Gamma(s)$ is the logarithmic derivative of the gamma function. 
Proof. First, we show that the height has the form

$$
h(M)=Z^{\prime}(M, 0)=A_{n}+B_{n} k_{n}(Q),
$$

for some constants $A_{n}$ and $B_{n}$. Since $Z(M, s)=(2 \pi)^{-2 s} Z_{Q^{*}}(s)$ by (2.3), it suffices to show that

$$
Z_{Q^{*}}^{\prime}(0)=A_{n}^{\prime}+B_{n}^{\prime} k_{n}(Q),
$$

for some constants $A_{n}^{\prime}$ and $B_{n}^{\prime}$. Applying the functional equation in Theorem 2.1(ii),

$$
Z_{Q^{*}}(s)=\pi^{2 s-n / 2} \frac{\Gamma(n / 2-s)}{\Gamma(s)} Z_{Q}(n / 2-s) .
$$

On the other hand, $Z_{Q}$ has meromorphic continuation, so it may be written as

$$
Z_{Q}(w)=\frac{a_{-1}}{w-n / 2}+a_{0}+O(w-n / 2)
$$

or setting $w=n / 2-s$,

$$
Z_{Q}(n / 2-s)=\frac{-a_{-1}}{s}+a_{0}+O(s),
$$

where $a_{0}$ is just $k_{n}(Q)$.

Substituting into (2.5),

$$
Z_{Q^{*}}(s)=\pi^{2 s-n / 2} \frac{\Gamma(n / 2-s)}{\Gamma(s)}\left\{\frac{-a_{-1}}{s}+k_{n}(Q)+O(s)\right\} .
$$

Because $\Gamma(s)$ has a simple pole at $s=0$,

$$
=s f(s)\left\{\frac{-a_{-1}}{s}+k_{n}(Q)+O(s)\right\},
$$

for some function $f(s)=b_{0}+b_{1} s+O\left(s^{2}\right)$ that is analytic at $s=0$,

$$
\begin{aligned}
& =s\left(b_{0}+b_{1} s+O\left(s^{2}\right)\right)\left\{\frac{-a_{-1}}{s}+k_{n}(Q)+O(s)\right\} \\
& =-b_{0} a_{-1}+\left(-b_{1} a_{-1}+b_{0} k_{n}(Q)\right) s+O\left(s^{2}\right) .
\end{aligned}
$$

Differentiating,

$$
Z_{Q^{*}}^{\prime}(0)=-b_{1} a_{-1}+b_{0} k_{n}(Q) .
$$

Computing the constants is a straightforward exercise; their values are:

$$
\begin{gathered}
a_{-1}=\pi^{n / 2} \Gamma(n / 2)^{-1}, \\
b_{0}=1 / a_{-1}, \\
b_{1}=\pi^{-n / 2} \Gamma(n / 2)\{2 \log \pi-\psi(n / 2)-\gamma\},
\end{gathered}
$$

where $\gamma$ is Euler's constant. From these we obtain the values of $A_{n}$ and $B_{n}$ in the theorem. 


\section{EXISTENCE OF EXTREMA}

In this section, we prove the following existence theorem:

Theorem 3.1. The height on the moduli space of n-dimensional flat tori of volume 1 attains a minimum.

Rather than using the explicit formula of Theorem 2.3 - which is more suitable for computational purposes - we express the Epstein zeta function in another form via an integral representation. This is an old technique due to Riemann, see [Te1].

Lemma 3.2 (Riemann). The Epstein zeta function $Z_{Q}(s)$ may be expressed as

$$
\begin{aligned}
\pi^{-s} \Gamma(s) Z_{Q}(s)= & \frac{|Q|^{-1 / 2}}{s-n / 2}-\frac{1}{s} \\
& \quad+\sum_{a \in \mathbf{Z}^{n}-0}\left(\int_{1}^{\infty} e^{-\pi Q[a] t} t^{s} \frac{d t}{t}+\int_{1}^{\infty} e^{-\pi Q^{-1}[a] t} t^{n / 2-s} \frac{d t}{t}\right) .
\end{aligned}
$$

Proof. (Theorem 3.1) We view the moduli space of flat tori as the space of lattices $\mathbf{L}^{n}$ described in section 1 . Given a lattice $L \in \mathbf{L}^{n}$, its minimal vector $m_{L}$ is defined to be the vector $x \in \mathbf{Z}^{n}$ that minimizes its associated quadratic form $Q[x]$. A set of lattices whose minimal vector is of bounded length has compact closure, so on such a set the height attains a minimum. Therefore, it suffices to show that the height becomes arbitrarily large for lattices whose minimal vector is sufficiently short.

Now the volume of the lattices is normalized to 1 , so $\left|m_{L}^{*}\right| \rightarrow 0$ as $\left|m_{L}\right| \rightarrow 0$. Hence by (2.3), all we need to prove is that $Z_{Q^{*}}^{\prime}(0) \rightarrow+\infty$ as $\left|m_{L}^{*}\right| \rightarrow 0$.

By Lemma 3.2,

$$
\begin{aligned}
Z_{Q^{*}}(s)= & Z_{Q^{-1}}(s)=\frac{\pi^{s}}{\Gamma(s)}\left(\frac{1}{s-n / 2}-\frac{1}{s}\right) \\
& +\frac{\pi^{s}}{\Gamma(s)}\left\{\sum_{a \in \mathbf{Z}^{n}-0}\left(\int_{1}^{\infty} e^{-\pi Q^{-1}[a] t} t^{s} \frac{d t}{t}+\int_{1}^{\infty} e^{-\pi Q[a] t} t^{n / 2-s} \frac{d t}{t}\right)\right\} .
\end{aligned}
$$

At $\left.\frac{d}{d t}\right|_{s=0}$, the first term is analytic at $s=0$ and independent of $Q$, so it contributes a constant $C$. Let $F_{Q}(s)$ denote the function in the braces. Then

$$
\left.\frac{d}{d s} Z_{Q^{*}}(s)\right|_{s=0}=C+\left[\frac{\pi^{s}}{\Gamma(s)} F_{Q}^{\prime}(s)+\frac{\pi^{s} \log \pi}{\Gamma(s)} F_{Q}(s)+\frac{\pi^{s}}{\Gamma(s)}\left(\frac{-\Gamma^{\prime}(s)}{\Gamma(s)}\right) F_{Q}(s)\right]_{s=0} .
$$

Because $F_{Q}(0)$ and $F_{Q}^{\prime}(0)$ clearly converge and $1 / \Gamma(0)=0$, the second and third terms vanish. In the last term, the factor $\frac{\pi^{s}}{\Gamma(s)}\left(\frac{-\Gamma^{\prime}(s)}{\Gamma(s)}\right)$ is a positive real number. Hence it suffices to check that $F_{Q}(0)$ tends to $+\infty$ as the shortest vector tends to zero.

By positivity of the terms,

$$
\begin{aligned}
F_{Q}(0) & =\sum_{a \in \mathbf{Z}^{n}-0} \int_{1}^{\infty} e^{-\pi Q^{-1}[a] t} \frac{d t}{t}+\sum_{a \in \mathbf{Z}^{n}-0} \int_{1}^{\infty} e^{-\pi Q[a] t} t^{n / 2} \frac{d t}{t} \\
& \geq \int_{1}^{\infty} \sum_{a \in \mathbf{Z}^{n}-0} e^{-\pi Q^{-1}[a] t} \frac{d t}{t} .
\end{aligned}
$$


Now let $r$ be the length of the minimal vector of the lattice $L^{*}$, and fix $T>1$. Discarding all but multiples of the shortest vector, the last integral is

$$
\begin{aligned}
& \geq \int_{1}^{T} \sum_{n \geq 1} e^{-\pi(r n)^{2} t} \frac{d t}{t} \\
& \geq \int_{1}^{T} \int_{1}^{\infty} e^{-\pi t(r x)^{2}} d x \frac{d t}{t} \\
& \geq \int_{1}^{T} \int_{r \sqrt{\pi t}}^{\infty} e^{-u^{2}} \frac{d u}{r \sqrt{\pi t}} \frac{d t}{t} .
\end{aligned}
$$

Let $B$ be the constant defined by $\int_{T}^{\infty} e^{-t^{2}} d t=B \sqrt{\pi}$, then as soon as $r<\sqrt{T / \pi}$ and upon integrating, we obtain

$$
=\frac{2 B}{r}\left(1-\frac{1}{\sqrt{T}}\right) \text {. }
$$

And this goes to $+\infty$ as $r \rightarrow 0$.

\section{Some Results on the EXtrema}

In this section we give some results on the extrema of the height and $Z_{Q}(s)$ for $n=2$ and $n=3$, and conclude with a couple of conjectures for the general case.

Let $L_{0}^{n}$ denote the lattice in $\mathbf{L}^{n}$ with the longest minimal vector, and denote its quadratic form by $Q_{0}^{n}$. It is well known that $L_{0}^{2}$ is the hexagonal lattice with basis matrix

$$
\left(\begin{array}{cc}
\sqrt{3} / 2 & 1 / 2 \\
0 & 1
\end{array}\right)
$$

and $L_{0}^{3}$ is the face-centered cubic lattice with basis matrix

$$
\left(\begin{array}{ccc}
1 / \sqrt{2} & 1 / 2 & 1 / 2 \\
0 & 1 & 0 \\
0 & 0 & 1
\end{array}\right) .
$$

These distinguished lattices possess a number of interesting properties; among them is that they provide the densest lattice packing of spheres in $\mathbf{R}^{2}$ and $\mathbf{R}^{3}$, respectively. See Conway and Sloane [CS].

For $n=2$, by considering the properties of the Dedekind eta-function in Kronecker's Limit Formula in (2.1), Osgood-Phillips-Sarnak [OPS] arrived at the following result:

Theorem 4.1 ([OPS]). The height on the moduli space of 2-d flat tori of area 1 has a global minimum at the torus corresponding to the hexagonal lattice.

Independent and earlier work on minimizing the Epstein zeta-function began by Rankin [Ra] and carried further by Cassels [Ca], Ennola [En1] and Diananda [Di] produced the following result which encompasses Theorem 4.1.

Theorem 4.2 (CDER). Let $Q$ be a 2-d positive definite quadratic form with determinant 1 . Then for $s$ with $\operatorname{Re}(s)>0$,

$$
Z_{Q}(S)-Z_{Q_{0}^{2}}(S) \geq 0
$$

and equality holds iff $Q$ is the quadratic form $Q_{0}^{2}$ of the hexagonal lattice. 
By the definition of $k_{n}(z)$ in (2.4), it is clear that at the point $s=1$, Theorem 4.2 is equivalent to Theorem 4.1 .

For $n=3$, Ennola [En2] managed a partial generalization of Theorem 4.2.

Theorem 4.3 (Ennola). Let $Q$ be a 3-d positive definite quadratic form with determinant 1 . Then for $s$ with $\operatorname{Re}(s)>0$, and $Q$ in a sufficiently small neighborhood of $Q_{0}^{3}$,

$$
Z_{Q}(S)-Z_{Q_{0}^{3}}(S) \geq 0
$$

and equality holds iff $Q$ is the quadratic form $Q_{0}^{3}$ of the face-centered cubic lattice.

Again, at the pole $s=3 / 2$, this is equivalent to the height having a local minimum.

Corollary 4.4. The height on the moduli space of 3-d flat tori of volume 1 has a local minimum at the torus corresponding to the face-centered cubic lattice.

The author has checked the height of $L_{0}^{3}$ against a set of points that are equidistributed in $\mathbf{L}^{3}$. The Hecke points provide such a set, see Chiu [Ch]. For $n=3$ and $p$ prime, the Hecke points are

$$
S(p)=\left\{\left(\begin{array}{ccc}
p & & \\
& 1 & \\
& & 1
\end{array}\right),\left(\begin{array}{ccc}
1 & a & \\
& p & \\
& & 1
\end{array}\right),\left(\begin{array}{lll}
1 & & a \\
& 1 & b \\
& & p
\end{array}\right) \mid 0 \leq a, b<p\right\} .
$$

For $S(101)$, which has 10,303 points (some of which have the same height value due to symmetries), the height as computed using formula (2.2) is strictly greater than the height of $L_{0}^{3}$.

We conclude with some general conjectures on the minimum of the height and the Epstein zeta-function. Heuristically, from examining the sum

$$
Z_{Q}(s)=\sum_{x \in \mathbf{Z}^{n}}^{\prime} Q[x]^{-s}
$$

it is apparent that the shorter vectors play a greater role, so it is reasonable to speculate that the quadratic form of lattices with longer minimal vectors would have smaller values for $Z_{Q}(s)$ for fixed $s$. This should certainly be the case for large values of $|s|$, and a result of Ryshkov [Ry] confirms it. For small values of $|s|$, the question becomes more delicate and is yet to be resolved. In light of the results in this section, we state the following conjectures on the height and $Z_{Q}(s)$, which we attribute to Sarnak and Rankin, respectively.

Conjecture 4.5 (Sarnak). The height on the moduli space of $n$-d flat tori of volume 1 has a global minimum at the torus corresponding to the lattice with the longest minimal vector.

Conjecture 4.6 (Rankin). Let $Q$ be an $n$-d positive definite quadratic form with determinant 1. Then for $s$ with $\operatorname{Re}(s)>0$,

$$
Z_{Q}(S)-Z_{Q_{0}^{n}}(S) \geq 0
$$

and equality holds iff $Q$ is the quadratic form $Q_{0}^{n}$ of the lattice with the longest minimal vector. 


\section{REFERENCES}

[Ca] J. W. S. Cassels, On a problem of Rankin about the Epstein zeta function, Proc. Glasgow Math. Assoc. 4 (1959), 73-80; 6 (1963), 116. MR 22:7975; MR 27:4803

[Ch] P. Chiu, Covering with Hecke points, J. Number Theory 53 (1995), 25-44. CMP 95:16

[CS] J. H. Conway and N. J. A. Sloane, Sphere Packings, Lattices, and Groups, Springer-Verlag, 1988. MR 89a: 11067

[Di] P. H. Diananda, Notes on two lemmas concerning the Epstein zeta-function, Proc. Glasgow Math. Assoc. 6 (1964), 202-204. MR 29:5798b

[Ef] I. Efrat, On a $G L(3)$ analog of $|\eta(z)|$, J. Number Theory 40 (1992), 174-186. MR 93a:11040

[En1] V. Ennola, A lemma about the Epstein zeta function, Proc. Glasgow Math. Assoc. 6 (1964), 198-201. MR 29:5798a

[En2] , On a problem about the Epstein zeta function, Proc. Cambridge Philos. Soc. 60 (1964), 855-875. MR 29:5797

[OPS] B. Osgood, R. Phillips, and P. Sarnak, Extremals of determinants of Laplacians, J. Funct. Analysis 80 (1988), 148-211. MR 90d:58159

[Ra] R. A. Rankin, A minimum problem for the Epstein zeta function, Proc. Glasgow Math. Assoc. 1 (1953), 149-158. MR 15:507c

[Ry] S. S. Ryshkov, On the question of final $\zeta$-optimality of lattices providing the closest lattice packing of $n$-dimensional spheres, Sibirsk. Mat. Zh. 14 (1973), 1065-1075; English transl., Siberian Math. J. 14 (1973), 743-750.

[Sa] P. Sarnak, Determinants of Laplacians; height and finiteness, Analysis et cetera, ed. P. Rabinowitz and E. Zehnder, Academic Press, 1990, pp. 601-622. MR 91d:58260

[Te1] A. Terras, Harmonic Analysis on Symmetric Spaces and Applications, Vol. I, SpringerVerlag, 1985. MR 87f:22010

[Te2] - Bessel series expansions of the Epstein zeta function and the functional equation, Trans. Amer. Math. Soc. 183 (1973), 477-486. MR 48:2091

P.O. Box 7486, Palo Alto, California 94309 